\title{
Compact optical trapping microscope using a diode laser
}

\section{Z Ulanowski and I K Ludlow}

Department of Physical Sciences, University of Hertfordshire, Hatfield, Herts AL10 9AB, UK.

E-mail: z.ulanowski@herts.ac.uk

\begin{abstract}
Single beam gradient force optical trapping (laser tweezers) is increasing in importance as a technique for microparticle and cell manipulation and for the measurement of piconewton forces in aqueous media. An optical trap constructed using an optical microscope and a diode laser is described here. The system incorporates novel beam steering optics allowing the positioning of the trapping spot within the field of view of the microscope. Despite its full functionality the system is compact, portable, relatively inexpensive, and easy to construct and operate. A new scheme for trapping force calibration is also reported and measurements compared to theoretical results. The trapping efficiency is found to be similar to that obtained when using other trapping systems.
\end{abstract}

Key words: optical trapping, laser tweezers, diode laser, trapping efficiency 


\section{Introduction}

Single beam optical trapping, often called laser tweezers, is a non-invasive technique for the manipulation of microparticles by using electromagnetic forces present in a strongly focused laser beam (Ashkin et al. 1986). Such forces arise from radiation pressure near the focus of the beam so as to produce trapping longitudinal and transverse with respect to the beam axis. It was demonstrated early on that living cells (Ashkin et al. 1987) and even organelles within intact cells (Ashkin et al. 1990) may be held by near infrared laser beams without sustaining damage. When combined with an imaging device such as an optical microscope, the technique offers the user a highly selective means of choosing a particle and transporting it to another location. There is now considerable interest in exploiting optical trapping for particle separation, transfer and concentration over fields as diverse as biology, medicine, materials science or physical chemistry. Some of the most promising application areas are in biosciences, where the technique can be used for cell, organelle, membrane and macromolecule trapping, isolation, sorting and manipulation.

Optical trapping is also growing in importance as a quantitative tool - for measuring small forces and torques, or as an essential "enabling" technique assisting a measurement process. In all these cases the interaction can be very selective - involving single molecules in some circumstances, as in the case DNA or mechanoenzymes attached to microspheres acting as "handles" (Perkins et al. 1994). In specific areas, such as the study of mechanoenzymes where the measurement of piconewton forces generated by "motor molecules" is necessary, optical trapping has already matured into a nearly indispensable research tool (Simmons et al. 1996, Mehta et al. 1998). Situations for which optical traps are uniquely suited occur where a microscopic object has to be maintained in isolation from solid supports, like in light scattering, spectroscopy and related measurements of microparticles, droplets, bubbles or other fragile structures such as micelles (Doornbos et al. 1996, Urlaub et al. 1996, Grier 1997, Gensch et al. 1998). Further areas of application include: cell-cell interactions, membrane properties (viscoelasticity, lateral diffusion etc.), cellular transport and the cytoskeleton (e.g. chromosome movement during cell division), cell motility, assisting cell microsurgery (e.g. chromosome dissection), cell fusion in genetic engineering, cell attachment to surfaces, scanning probe microscopy, micromachining - for reviews see Hoffmann (1996), Ashkin (1997), Khan and Sheetz (1997), Sheetz (1998). Consequently, optical trapping can be expected to become a routine measurement and manipulation technique in many varied applications within research and analytic laboratories.

There are also prospects for the application of optical trapping as a general-purpose tool which can change passive observation using an optical microscope into a more active, manipulative examination, possibly combined with some degree of quantitative force transduction. Examination of many types of microscopic specimens can be aided by the ability to rearrange and orientate objects either in one plane or in three dimensions. An optical trap makes viewing an object from more than one direction quite easy. It also allows testing particle attachment to surfaces, bringing objects (such as cells of different types) into contact, controlled deposition of microscopic objects (for example labelled microbeads or micromachined particles), manipulation of organelles within intact cells and immobilisation of motile cells. The potential for use of optical trapping in education, for instance in demonstrations taking advantage of video microscopy, is also significant 
but it remains largely unexplored (Ulanowski et al. 1996). Currently, one of the main barriers preventing broader application of optical trapping is the cost of necessary equipment.

Optical traps using diode lasers have been in use in the authors' laboratory since 1988 (Ulanowski 1993). While one factor behind the development of a diode laser based system was cost, the motivation was to create an instrument which was compact, portable, comparatively rugged, and one that could be used by non-specialists. Therefore, user friendliness and freedom from adjustment were also necessary prerequisites. A further requirement was dictated by a specific need for a system for use in single-particle, multi-angle light scattering measurements, also known as laser diffractometry (Ulanowski et al. 1987, Doornbos et al. 1996), where light is detected in a semi-circle surrounding the sample, implying that good access to the trapping area must exist. For practical reasons, it is advantageous to use an inverted microscope configuration in this context. However, instead of employing an unwieldy and expensive purpose-built inverted microscope, a trap can be constructed using an upright microscope capable of being operated in either orientation.

This paper describes such a low cost but easy to use and portable system. The trap is constructed using an optical microscope with a diode laser forming its integral part. The system incorporates beam steering optics allowing the positioning of the laser beam spot within the field of view of the microscope. An unusual feature of the design is that beam scanning is achieved by displacing the laser in the plane perpendicular to the laser beam. Such an arrangement does not appear to have previously been used in the context of optical trapping or, indeed, optical microscopy in general. Trapping force measurements using a new scheme are also reported.

\section{System description}

The laser trap was constructed by incorporating a laser into a microscope whilst retaining its imaging capability. The system includes an infra-red diode laser and motor-driven beam steering optics which can be controlled manually (e.g. using a computer mouse) or by a computer. The steering is accomplished by displacing the laser with relation to the microscope using linear slides. The position of the trapping spot can be varied with submicrometer resolution. Observation of trapped particles and of the beam spot is made possible by a CCD camera attached to the microscope (figure 1).

\subsection{Optical system}

Operation of optical traps depends on the ability to focus light into a very small spot, almost invariably using a high numerical aperture lens. In turn, efficient concentration of light requires the utilisation of the whole aperture of the focusing lens. While exact computation of the properties of a beam focused by a high numerical aperture lens is not possible at present, sufficient insight can be gained using approximate methods. In the case of a Gaussian beam in the paraxial regime the size $D$ of the spot is: 
where

$\lambda$ - wavelength of light,

$f$ - focal length of the lens,

$n$ - refractive index of the medium (water in this case),

$\phi$ - beam diameter at the lens.

When the beam diameter becomes much greater than the clear aperture of the lens the following approximate formula applies:

where the beam diameter $\phi$ is now, emphatically, the same as the lens diameter and it is assumed that, for comparison with formula (1), the spot diameter is measured between the $1 / \mathrm{e}^{2}$ irradiance levels. It should be noted that direct comparison between the formulae (1) and (2) is misleading: the former assumes total absence of beam truncation, the latter a "top hat" profile. Since a beam much narrower than the lens aperture would not produce a spot of the minimum size and a wider beam would result in a loss of optical power, conflicting demands must be met when selecting the parameters of the optical system. A useful practical criterion for beam diameter selection can be to maximize the irradiance at the centre of the spot. In such a case, diffraction theory (Richards and Wolf 1959) shows that the optimum is achieved when the ratio of the beam to lens diameters is about 0.9 for a high numerical aperture lens. However, geometrical optics considerations indicate that longitudinal trapping forces (i.e. forces along the axis of the beam) increase as incident light is distributed away from the beam axis (Ashkin 1992). Therefore, if longitudinal rather than transverse forces are to be maximized, higher degree of overfilling may be beneficial.

The optical system will now be described with reference to figure 2 . The additional optics for producing a focused beam spot $\mathbf{S}$ in the object plane of the microscope objective lens MO comprises a laser producing a collimated beam, a focusing lens $\mathbf{L 1}$ and a coupling lens $\mathbf{L 2}$. The lens $\mathbf{L 1}$ is movable together with the laser in a plane perpendicular to its axis so as to displace the beam spot $\mathbf{S}$ in the object plane of the objective lens. L1 may additionally be made capable of limited movement parallel to its axis so that the beam spot $\mathbf{S}$ can be displaced a short distance away from the object plane of the objective lens. The mirror M1 separates visible light used for viewing the specimen and infra-red light emitted by the laser. A low wavefront-distortion "hot mirror" (dichroic beam splitter) designed for operation at $45^{\circ}$ angle of incidence can be used for this purpose. Visualising the beam spot is made possible by attaching a video camera to the microscope either as a sole viewing means or while retaining the original viewing optics; in the latter case a semi-transparent mirror or prism can provide the required image splitting (Ulanowski 1993).

As was pointed out earlier, in order to efficiently focus light into a small spot the whole clear aperture of the lens should be utilized. In the present system the requirements of minimizing the spot size while maintaining maximum optical throughput in the system are met by ensuring that, regardless of the position of the focused spot, (a) the beam is expanded to fill the clear aperture of the objective lens, and (b) the central ray is aimed at the principal point of the objective. Focusing the spot on the specimen under examination is achieved by arranging the focal point $\mathbf{F}$ of the focusing lens $\mathbf{L} \mathbf{1}$ in the plane of the image of the specimen as obtained through the objective and the coupling lens $\mathbf{L 2}$. This 
has also the effect of making a parallel laser beam divergent so as to adapt it to a microscope objective that is characterized by a finite conjugate ratio (finite tube length). This last point cannot be overemphasized, as optical trapping demands that optical aberrations be kept to a minimum and high numerical aperture microscope objectives are very sensitive to the placement of the conjugate image and object planes, as embodied by the microscope "tube length" parameter (the mechanical tube length being the distance between the objective and the eyepiece, measured along the optical path). Efficient trapping cannot be expected if a parallel beam is directed through a typical microscope objective (with the exception of "infinity optics" objectives). Moreover, the optimum tube length for trapping is likely to be different from the one for which the given objective was designed if infrared light used. If the difference is significant and trapping is impaired at normal tube length, a supplementary "tube lens" can be inserted between the dichroic mirror and the camera to alter the optical tube length while maintaining the mechanical tube length.

The main features of the optical system can thus be summarized as follows.

1. The focal point $\mathbf{F}$ of the focusing lens $\mathbf{L 1}$ is in the plane of the image of the specimen in the microscope objective lens MO and the coupling lens L2. If the lens L1 is positive, $\mathbf{F}$ is on the side of $\mathbf{L} \mathbf{1}$ facing the objective; if $\mathbf{L} \mathbf{1}$ is negative, $\mathbf{F}$ (virtual) is on the laser side.

2. The light beam fills the whole aperture of the microscope objective, irrespectively of the deflection of the beam. This is achieved by ensuring that the rear focal plane of the coupling lens $\mathbf{L} \mathbf{2}$ is close to the primary principal point of the microscope objective.

3. The laser and the focusing lens $\mathbf{L 1}$ assembly can be moved as a unit in the plane perpendicular to the optical axis of the assembly whereby the beam spot $\mathbf{S}$ moves within the object plane by an amount proportional to the displacement of the assembly, the demagnification being equal to the ratio of the focal lengths of the lenses MO and L2. If desired, the assembly can also be made capable of movement parallel to the axis, resulting in a respective displacement of the beam spot $\mathbf{S}$ away from the object plane, without any change in the diameter of the beam at the objective (degree of overfilling).

Formulae for the optical parameters of the system will now be obtained. It is assumed throughout that all distances are measured between appropriate principal points of the lenses. The diameter $\phi_{2}$ of the laser beam in the plane of the coupling lens $\mathbf{L} \mathbf{2}$ is described by:

where:

$\phi_{1}$ - original diameter of the collimated laser beam (i.e. near the lens L1),

$d$ - separation of the lenses $\mathbf{L} 1$ and $\mathbf{L 2}$,

$f_{1}$ - focal length of the lens $\mathbf{L 1}$ (negative for a negative power lens).

The virtual image of the focal point $\mathbf{F}$ in the lens $\mathbf{L} 2$ is formed at distance $l$ from the microscope objective lens MO (see figure 2). Because the distance between $\mathbf{L} 2$ and MO is equal to $f_{2}$, the lens equation for the lens $\mathbf{L} \mathbf{2}$ takes the form: 
The diameter $\phi$ of the beam in the plane of the microscope objective is:

By combining expressions (3), (4) and (5) we obtain the required equation:

The formula (6) allows choosing the focal lengths of the lenses given the collimated laser beam diameter and the diameter $\phi$ which is determined by the size of the clear aperture of the objective lens. The latter can be calculated with the aid of the formula

where $N$ and $f_{3}$ are, respectively, the numerical aperture and the focal length of the objective lens and $n$ is the refractive index of the immersion medium. It is evident from formula (6) that the beam diameter at the objective is independent of the position of the focusing lens $\mathbf{L 1}$ and, consequently, focused spot steering can be achieved without changing the degree of objective filling, ensuring that optimum conditions for trapping are maintained regardless of the position of the spot.

A natural starting point in the design process is the focal length $f_{2}$, which determines the distance between the coupling lens $\mathbf{L} \mathbf{2}$ and the objective lens. Having calculated $f_{1}$ from equation (6), one can obtain the distance $d$ between the lenses $\mathbf{L 1}$ and $\mathbf{L} 2$ from equation (4) (note again that $f_{1}$ can be positive or negative, depending on the type of the lens L1). The length $l$ is approximately equal to the object-to-image distance of the microscope (typically between 185 and $195 \mathrm{~mm}$ ) less the focal length $f_{3}$ and the principal point separation of the objective lens. It can be noted that the above formulae are also valid for the limiting case of "infinity optics" microscopes where the length $l$ is infinite.

\subsection{Microscope}

The trap was constructed using a Biolam S-20 upright microscope (LOMO, St Petersburg, Russia). This system was chosen because, somewhat unusually, it is focused by displacing the microscope limb together with the objective nosepiece, instead of the stage. Such a configuration is advantageous when vertical adjustment of the position of the trapping beam spot, rather than the specimen, is required (for example, in singleparticle light scattering measurements). The microscope was equipped with a LOMO OM-25 water immersion objective with a numerical aperture $\mathrm{NA}=1.23$ and a magnification of $\times 70$, as well as a more usual oil immersion lens. The advantage of the water immersion lens was that it could be operated without a cover glass, in effect giving a long working distance of up to about $200 \mu \mathrm{m}$. This is important in applications such as light scattering or cell isolation. The cover glass thickness adjustment could be accomplished by using a built-in correction ring of the objective or by increasing the tube length of the microscope, or both. For some uses considered for the system, such as educational ones, it is also significant that the chosen objective, while being of sufficiently high quality, is a fraction of the cost of high-NA water immersion objectives of western European or Japanese manufacture. 


\subsection{Laser}

A variety of near infra-red, index guided, single transverse mode laser diodes with powers ranging from 2 to $150 \mathrm{~mW}$ have been successfully tested with the microscope, most notably including SDL-5300 and SDL-5400 series devices (SDL, Santa Barbara, CA). The measurements presented in this paper were carried out using a Philips CQL72 "collimator pen" with $20 \mathrm{~mW}$ maximum power and $820 \mathrm{~nm}$ wavelength (now superseded by CP78 series $780 \mathrm{~nm}$ diodes). This particular device, whilst being inexpensive, has a built-in collimator. The laser was connected to an adjustable driver operating in "constant current" mode (this prevents laser power changes due to optical feedback, which might take place in "constant power" mode). The laser mount incorporated a heatsink. The latter can be supplemented with a small fan, especially if the laser assembly is mounted within an enclosure - this should extend the lifetime of the laser if the device is operated near its maximum output power.

\subsection{Beam steering}

In order to allow movement of the focused spot, the laser was mounted on two crossed linear slides to which small stepper motors $\left(7.5^{\circ}\right.$ step) were attached. In the system described here the step size was $2.5 \mu \mathrm{m}$ in the plane of the slides or $0.1 \mu \mathrm{m}$ in terms of focused spot movement (if needed, these values can be lowered by more than an order of magnitude through the use of smaller step-size motors or a microstepping controller). The cost of the system can be significantly reduced by using manual micrometer-driven positioners instead of motorized ones but a disadvantage of this option is, apart from operator discomfort, that manual positioning may transmit vibrations to the body of the microscope and dislodge the trapped object. Movement along the axis of the beam, which is rarely required in practice, was made possible by the use of a manually adjustable draw tube (figure 1).

The stepper motors were operated by a custom interface controlled using a computer mouse or a tracker ball, both with so-called quadrature output allowing easy interfacing to digital circuits. The same interface could be connected to a computer to provide preprogrammed movement of the trapping spot. Microswitches limited the range of travel of the slides.

\section{Trapping force measurement and computation}

Longitudinal trapping forces were measured by lifting a particle and then reducing laser power until the particle was observed to fall out of the trap under the influence of gravity. Transverse trapping forces were determined by comparing them to viscous drag forces calculated using Stokes' law. A semi-automatic scheme was used where a trapped particle was lifted above the microscope slide and circulated at variable velocities around a rectangular path in the horizontal plane - see figure 3 . In each cycle the spot velocity was maintained at a pre-defined minimum velocity $v_{0}$, except during middle portions of two opposite sides (designated I and III in figure 3) of the rectangle, where the spot was accelerated up to a maximum velocity $v_{\mathrm{m}}=2 v_{0}$. At the beginning of section $\mathbf{I}$ the velocities were incremented in each cycle (typically by 5\%). In this way a succession of progressively increasing maximum velocities was produced, until particle escape was observed. The escape velocity was then assumed to be between the two most recent 
maximum velocity values and the trapping force calculated accordingly. The purpose of the constant velocity section following the acceleration phase (figure 3 ) was to allow the spot to move away from the particle after its escape so that re-entrapment did not occur; the intermediate velocity section allowed trapped particles to decelerate before the rightangle turn.

The forces produced in optical traps can be expressed in terms of a non-dimensional trapping efficiency $Q=F c / n P$, where $F$ is the trapping force, $P$ the beam power, $c$ the velocity of light and $n$ the refractive index of the medium (usually water). The beam power was measured by hanging a hemispherical water droplet from the objective and using an optical power meter with an integrating sphere detector head, as shown in figure 4 ; a correction was applied for the $2 \%$ reflection coefficient at the water-air interface. Trapping efficiency results obtained for several sizes of polystyrene latex particles are given in table 1 and figure 5, and the trapping efficiency as a function of direction of movement is shown in figure 6 . The asymmetry of the trapping forces can be compared with the asymmetry of the laser beam, as represented by its irradiance profile shown in figure 7 . The irradiance was measured prior to focusing by the microscope objective at the position of the objective's principal point. It should be noted that the dimensions of the focused spot can be expected to be approximately inversely proportional to the corresponding dimensions of the beam prior to focusing, if geometric aberrations of the objective are neglected - see expression (1).

The model used for the computation of trapping forces was the standard ray optics one (Ashkin 1992) except that a well-corrected lens satisfying the sine condition was assumed. In such a lens the equivalent refracting surface (principal surface) is a section of a sphere centered on the focal point (Richards and Wolf 1959, Levi 1968, Gu et al. 1997) - see figure 8. A consequence of this assumption is that tangents, used in the paraxial approximation, are replaced by sines in expressions relating to the focused ray angle. Overall, ray angles are reduced for intermediate ray positions within the aperture of the lens, leading to smaller longitudinal trapping forces ( $\mathrm{Gu}$ et al. 1997) and (somewhat paradoxically) larger transverse forces in comparison with the paraxial approximation. The beam irradiance profile for the computations was selected to approximate the measured one (as seen in figure 7) by using a sixth-power exponential function instead of the usual Gaussian one:

where $x$ and $y$ denote distances from the beam axis and $w_{x}$ and $w_{y}$ the respective semiaxes. A comparison of the Gaussian and the 6th-power functions with the measured irradiance profiles is shown in figure 9.

\section{Discussion}

An optical trapping system using a diode laser mounted on an optical microscope has been constructed. It employs an unusual beam deflection scheme relying on displacing the laser together with a lens. The system utilizes only two singlets to achieve the aims of laser beam expansion, steering and confocality. Spot positioning in two or three dimensions can be accomplished without any change in the diameter of the beam at the microscope objective (degree of aperture filling), ensuring that optimum trapping 
conditions are maintained. A further advantage of this optical configuration is its short optical path, leading to a compact system. The fact that only two singlets are required simplifies the design and construction and reduces power losses - in the present system the optical transmission measured between the collimated beam and the focused spot was $45 \%$, which is quite high. At the same time optical aberrations due to the additional components can in principle be kept below the diffraction limit (partly due to relatively long focal lengths in comparison with the short path).

The trapping efficiency measurements showed performance similar to that of previously described systems (Sato et al. 1991, Afzal et al. 1992, Escandon et al. 1994), including those using much larger and more costly neodymium lasers (Felgner et al. 1995) despite the simplicity and low cost of the apparatus. While the transverse trapping efficiencies fall short of those predicted by the ray optics model, it is not clear whether this is due to instrumental factors such as optical aberrations or due to deficiencies of the model (most notably the exclusion of scattering and diffraction effects). It is also interesting to observe that the ray optics model for a lens obeying the sine condition, while being physically more correct in terms of the far field description of the beam, leads to greater discrepancies with measured values of the transverse forces than the standard paraxial model.

The measured forces were characterized by good symmetry in the horizontal plane (figure 6) despite the non-circular beam profile of the laser which was used (figure 7). The symmetry is partly accounted for by the fact that, in the absence of beam shape effects, trapping forces are larger in the direction parallel to the direction of polarization. For diode lasers the latter is parallel to the longer axis of the (elliptical) focused spot and the lower field gradient in this direction partially compensates for the effect of polarization. For these reasons beam circularization (e.g. using an anamorphic prism pair) would not bring much advantage as long as the trapped particles remained large. However, diode lasers with greater beam ellipticity, for example some higher power devices, might not produce the same result. Also, the spatial symmetry of the forces cannot be expected to be maintained for particle diameters comparable or smaller than the size of the focused spot.

While the aim of the present study was to design a demonstration, general purpose instrument, the trapping microscope can be useful in a range of specialist applications. As it is compact and self-contained, it can be easily incorporated into other experimental systems, such as light scattering apparatus. It can also be used in different orientations, for example instead of a purpose-built inverted microscope. A shortcoming of the configuration presented here is that it precludes the use of fast scanning optics such as galvanometer mirrors or acousto-optic scanners. Consequently, certain force measuring schemes would be difficult to implement if fast time-response was required. However, this problem could in principle be overcome by the application of piezoelectric positioners.

In general, the small size and low cost of systems using laser diodes (Afzal et al. 1992, Bakker Schut et al. 1993, Escandon et al. 1994, Doornbos et al. 1996) makes them very attractive in optical trapping. In addition, laser diode power can be easily modulated, which can be an advantage in some applications. The fact that single transverse mode, index-guided quantum-well diodes, which are characterized by nearly diffraction-limited 
output and low astigmatism, can successfully be used in optical traps and provide high trapping efficiencies runs counter to the opinion expressed sometimes that laser diodes are not a good choice in this context because their beam quality is inferior to that of other lasers.

The trapping force measurement scheme presented here can be put into practice relatively easily. The trapping forces can be determined independently in different directions, allowing the investigation of factors such as beam polarization and shape. The measurements are characterized by good repeatability. Whereas particle acceleration is necessary, this does not lead to significant errors since the discrepancy due to inertia is several orders of magnitude smaller than the viscous drag itself. The error is also smaller than the centripetal force present in the scheme where the particle moves along a circular path (Afzal et al. 1992) instead of a rectangular one. In conclusion, the scheme offers a simple yet flexible and convenient way of determining trapping forces and efficiency.

\section{Acknowledgements}

The authors are indebted to John Kerr for efficient implementation of the mechanical design and many useful ideas, and to Marko Edzes for writing the ray optics computer program. This research was supported in part by the Engineering and Physical Sciences Research Council.

\section{References}

Afzal R S and Treacy E B 1992 Optical tweezers using a diode-laser Rev. Sci. Instrum. $632157-2163$

Ashkin A 1986 Observation of a single-beam gradient force optical trap for dielectric particles Opt. Lett. 11 288-290

Ashkin A, Dziedzic J M and Yamane T 1987 Optical trapping and manipulation of single cells using infrared-laser beams Nature 330 769-771

Ashkin A, Schutze K, Dziedzic J M, Euteneuer U and Schliwa M 1990 Force generation of organelle transport measured in vivo by an infrared laser trap Nature 348 346-348

Ashkin A 1992 Forces of a single-beam gradient laser trap on a dielectric sphere in the ray optics regime Biophys. J. 61 569-582

Ashkin A 1997 Optical trapping and manipulation of neutral particles using lasers Proc. Nat. Ac. Sci. USA 94 4853-4860

Bakker Schut TC, Schipper EF, de Grooth BG, Greve J 1993 Optical-trapping micromanipulation using $780 \mathrm{~nm}$ diode-lasers Opt. Lett. 18 447-9

Doornbos R M P, Schaeffer M, Hoekstra A G, Sloot P M A, de Grooth B G and Greve J 1996 Elastic light-scattering measurements of single biological cells in an optical trap Appl. Opt. 35 729-734

Escandon G J, Liu Y, Sonek G J and Berns M W 1994 Beam magnification and the efficiency of optical trapping with $790 \mathrm{~nm}$ AlGaAs laser diodes IEEE Photon. Technol. Lett. 6 597-600

Felgner H, Muller O, Schliwa M 1995 Calibration of light forces in optical tweezers Appl. Opt. 34 977-982

Gensch T, Hofkens J, van Stam J, Faes H, Creutz S, Tsuda K, Jerome R, Masuhara H and de Schryver F C 1998 Transmission and confocal fluorescence microscopy and 
time-resolved fluorescence spectroscopy combined with a laser trap J. Phys. Chem. B 102 8440-8451

Grier D G 1997 Optical tweezers in colloid and interface science Curr. Opinion Coll. Interface Sci. 2 264-270

Gu M, Ke P C and Gan X S 1997 Trapping force by a high numerical-aperture microscope objective obeying the sine condition Rev. Sci. Instrum. 68 3666-3668

Hoffmann F 1996 Laser microbeams for the manipulation of plant-cells and subcellular structures Plant Sci. 113 1-11

Khan S and Sheetz M P 1997 Force effects on biochemical kinetics Ann. Rev. Biochem. 66 785-805

Levi L 1968 Applied Optics (New York: Wiley) p 414

Mehta A D, Finer J T and Spudich J A 1998 Use of optical traps in single-molecule study of nonprocessive biological motors Meth. Enzymol. 298 436-459

Perkins T T, Smith D E and Chu S 1994 Direct observation of tube-like motion of a single polymer-chain Science 264 819-822

Richards B and Wolf E 1959 Electromagnetic diffraction in optical systems Proc. Roy. Soc. London A 253 358-379

Sato S, Ohyumi M, Shibata H, Inaba H and Ogawa Y 1991 Optical trapping of small particles using a $1.3 \mu \mathrm{m}$ compact InGaAsP diode-laser Opt. Lett. 16 282-284

Sheetz M P (ed.) 1998 Laser Tweezers in Cell Biology. Methods in Cell Biology 55 (San Diego: Academic Press)

Simmons R M, Finer J T, Chu S and Spudich J A 1996 Quantitative measurements of force and displacement using an optical trap Biophys. J. 70 1813-1822

Ulanowski Z, Ludlow I K and Waites W M 1987 Water-content and size of spore components determined by laser diffractometry FEMS Microbiol. Lett. 40 229-232

Ulanowski Z 1993 GB Patent 2,231,681; US Patent 5,225,929

Ulanowski Z and Williams I R 1996 Optical tweezers Phys. Education 31 179-182

Urlaub E, Lankers M, Kiefer W 1996 Applications of the optical trapping technique to analyze chemical reactions in single emulsion particles Fresenius J. Anal. Chem. 355 329-331 
Table 1. Mean measured and computed trapping efficiency for polystyrene spheres in four different directions, denoted by the subscripts $x, y, 45$ and $z$ : $\mathrm{x}$ is parallel to the short axis of the focused spot, $y$ is parallel to the long axis and the direction of polarization, 45 refers to the diagonal direction between the two and $z$ is parallel to the beam axis.

\begin{tabular}{|c|c|c|c|c|c|c|c|c|}
\hline \multirow[b]{2}{*}{$\begin{array}{l}\text { Diameter } \\
(\mu \mathrm{m})\end{array}$} & \multicolumn{2}{|c|}{$Q_{X}$} & \multicolumn{2}{|c|}{$Q_{Y}$} & \multicolumn{2}{|c|}{$Q_{45}$} & \multicolumn{2}{|c|}{$Q_{Z}$} \\
\hline & $\begin{array}{c}\text { measured } \\
(-)\end{array}$ & $\begin{array}{c}\text { computed } \\
(-)\end{array}$ & $\begin{array}{c}\text { measured } \\
(-)\end{array}$ & $\begin{array}{c}\text { computed } \\
(-)\end{array}$ & $\begin{array}{c}\text { measured } \\
(-)\end{array}$ & $\begin{array}{c}\text { computed } \\
(-)\end{array}$ & $\begin{array}{c}\text { measured } \\
(-)\end{array}$ & $\begin{array}{c}\text { computed } \\
(-)\end{array}$ \\
\hline 2.2 & 0.08 & 0.25 & 0.10 & 0.30 & 0.11 & 0.27 & - & - \\
\hline 5.3 & 0.11 & 0.25 & 0.11 & 0.31 & 0.14 & 0.27 & - & - \\
\hline 6.8 & 0.11 & 0.24 & 0.12 & 0.30 & 0.15 & 0.28 & - & - \\
\hline 9.6 & 0.11 & 0.24 & 0.12 & 0.28 & 0.13 & 0.26 & 0.098 & 0.106 \\
\hline
\end{tabular}

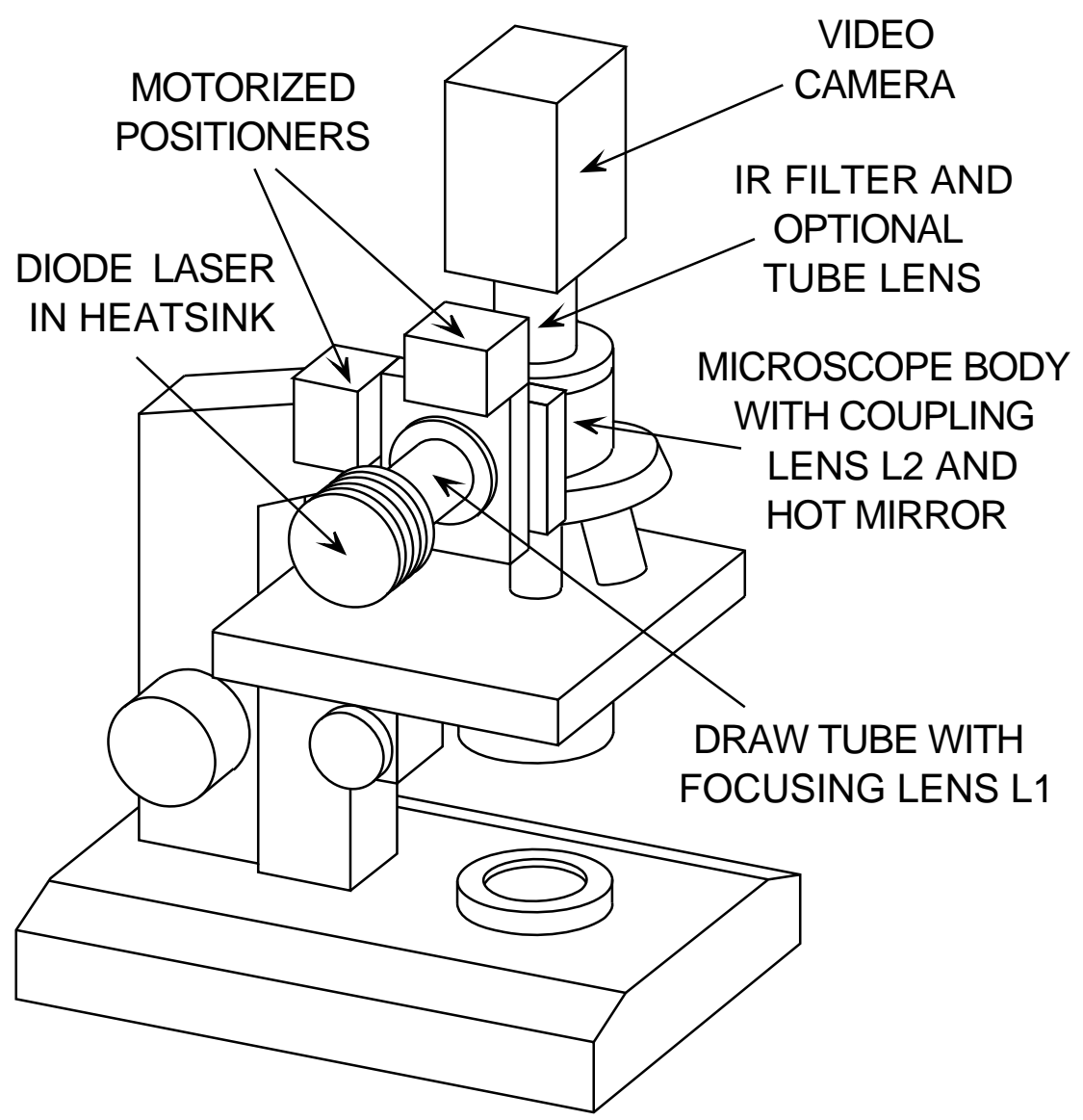

Figure 1. The trapping microscope. 


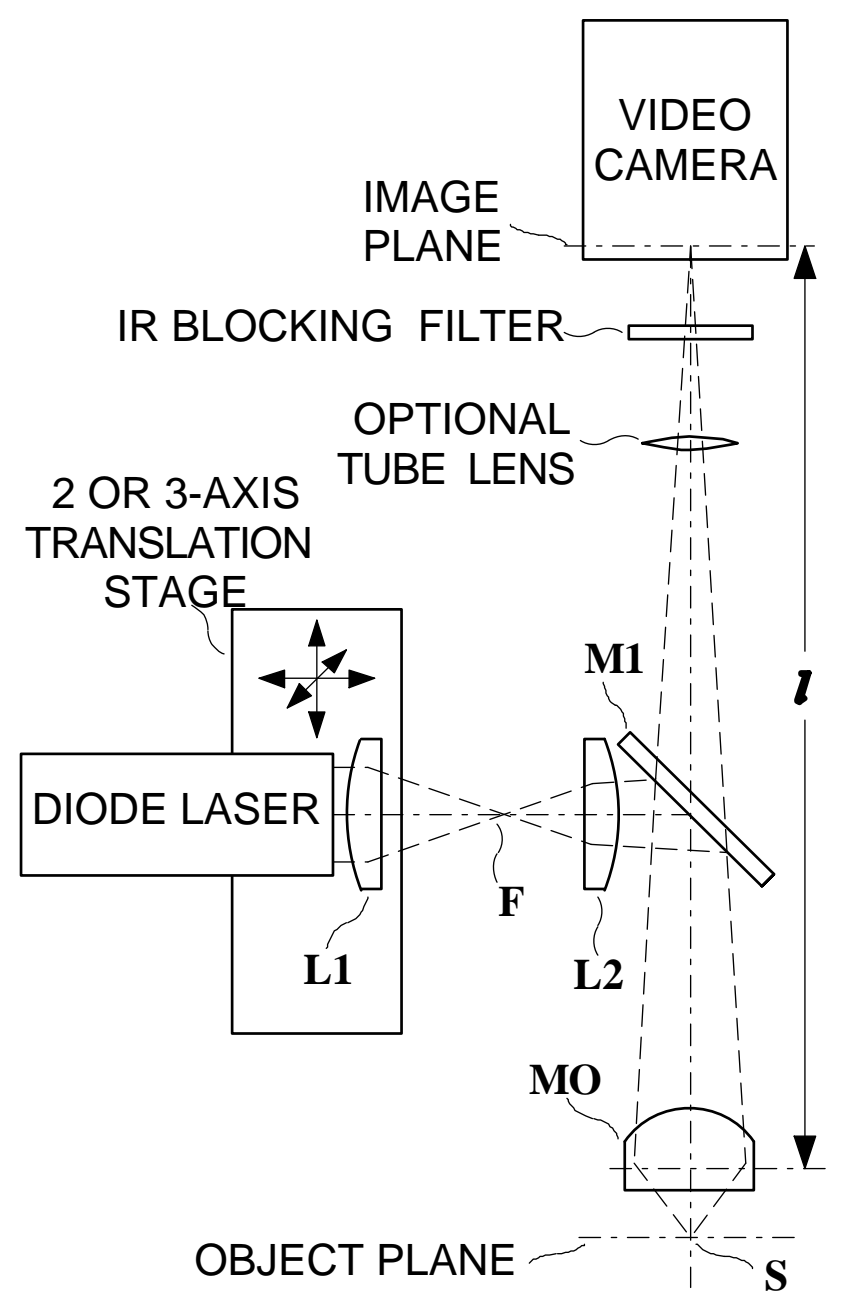

Figure 2. Optical system of the trapping microscope comprising: a diode laser, microscope objective lens MO, focused spot $\mathbf{S}$, focusing lens $\mathbf{L 1}$ with focal point $\mathbf{F}$, coupling lens $\mathbf{L 2}$, dichroic mirror M1.

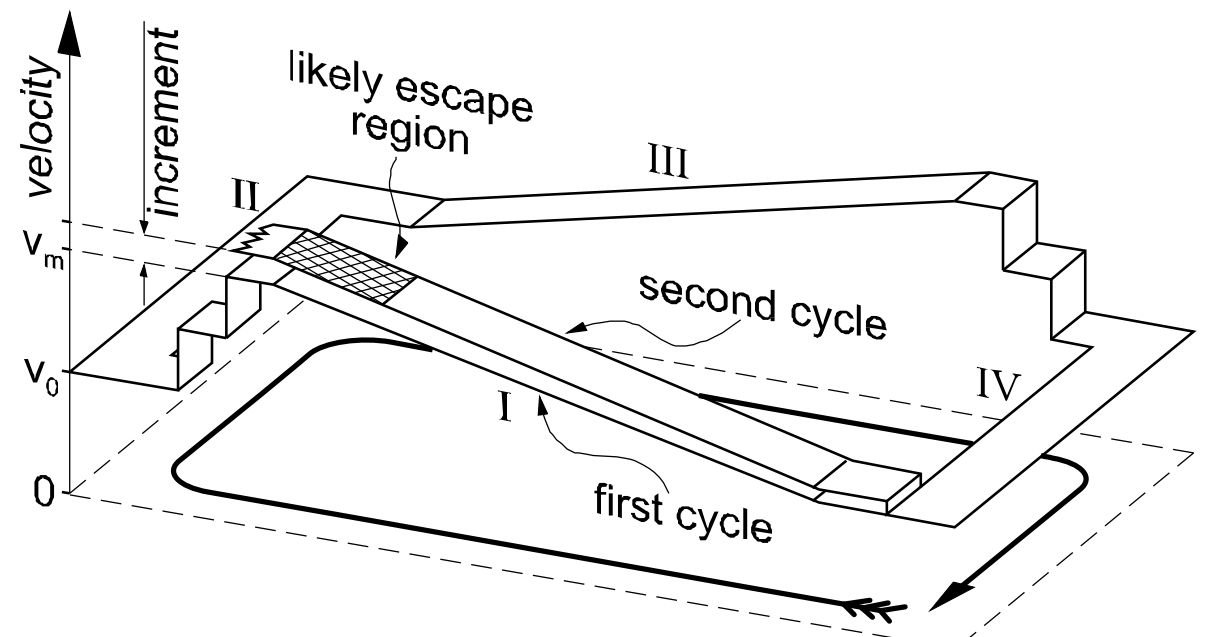

Figure 3. The path of the beam spot during escape velocity measurement showing velocity (vertical axis) as a function of position. 


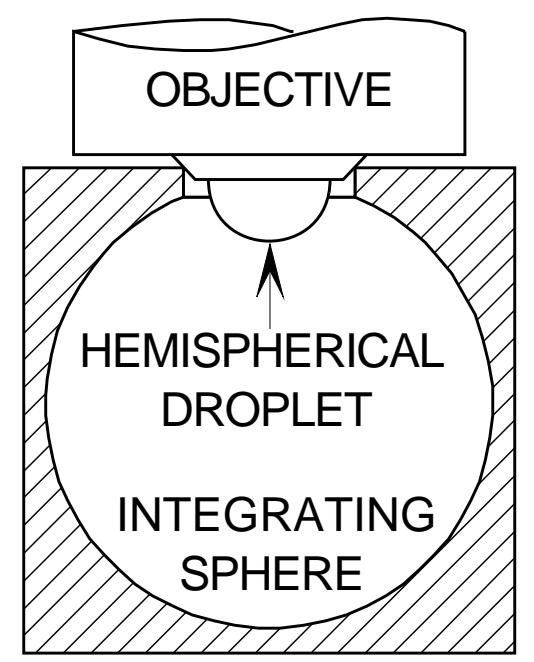

Figure 4. Focused beam power measurement for a water immersion objective.

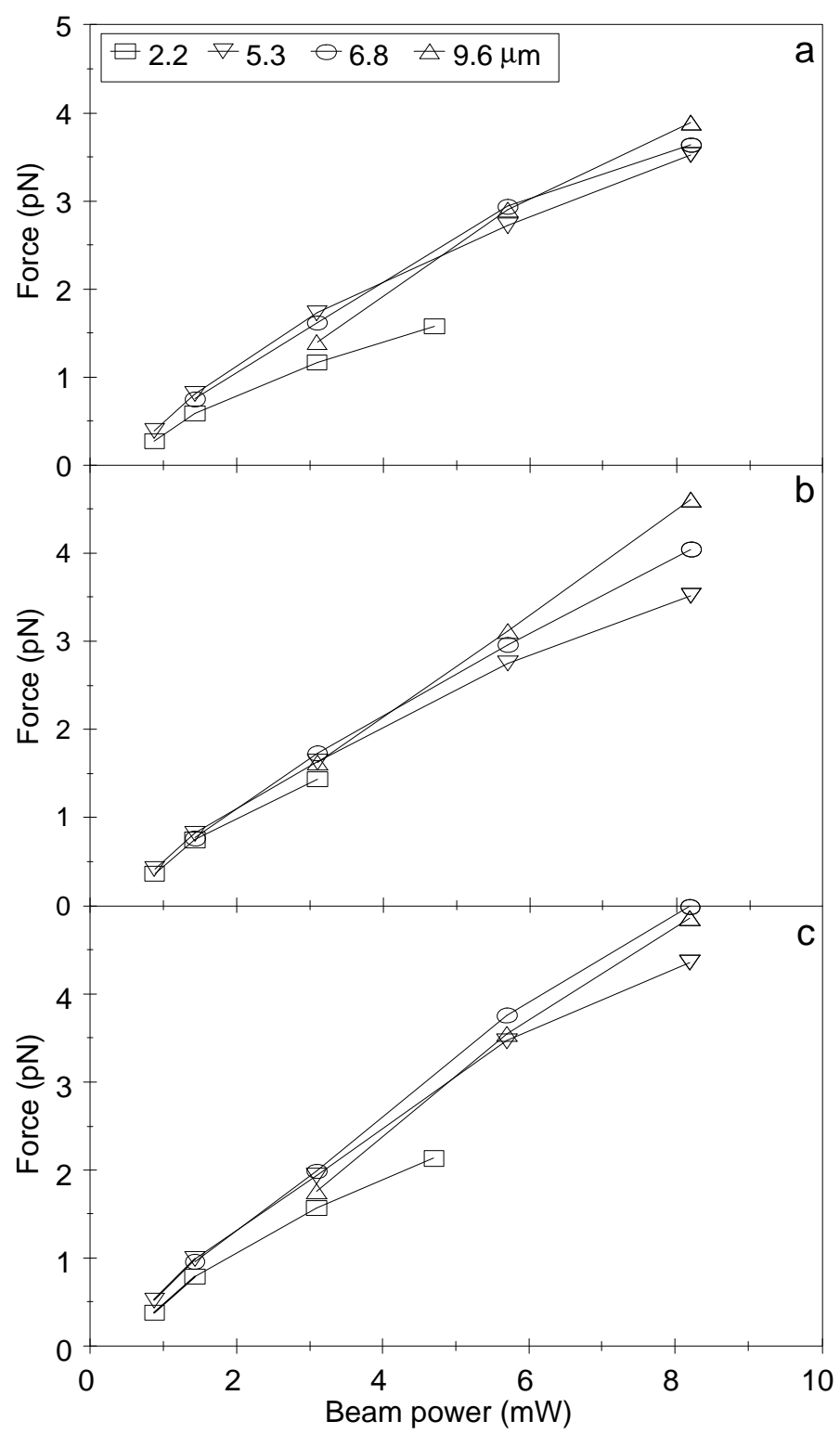

Figure 5. Transverse trapping force as a function of beam power for several polystyrene latex sphere sizes. The direction of movement is: a) parallel, b) perpendicular, and c) $45^{\circ}$ to the long axis of the beam prior to focusing. Beam power is measured at the focused spot. 

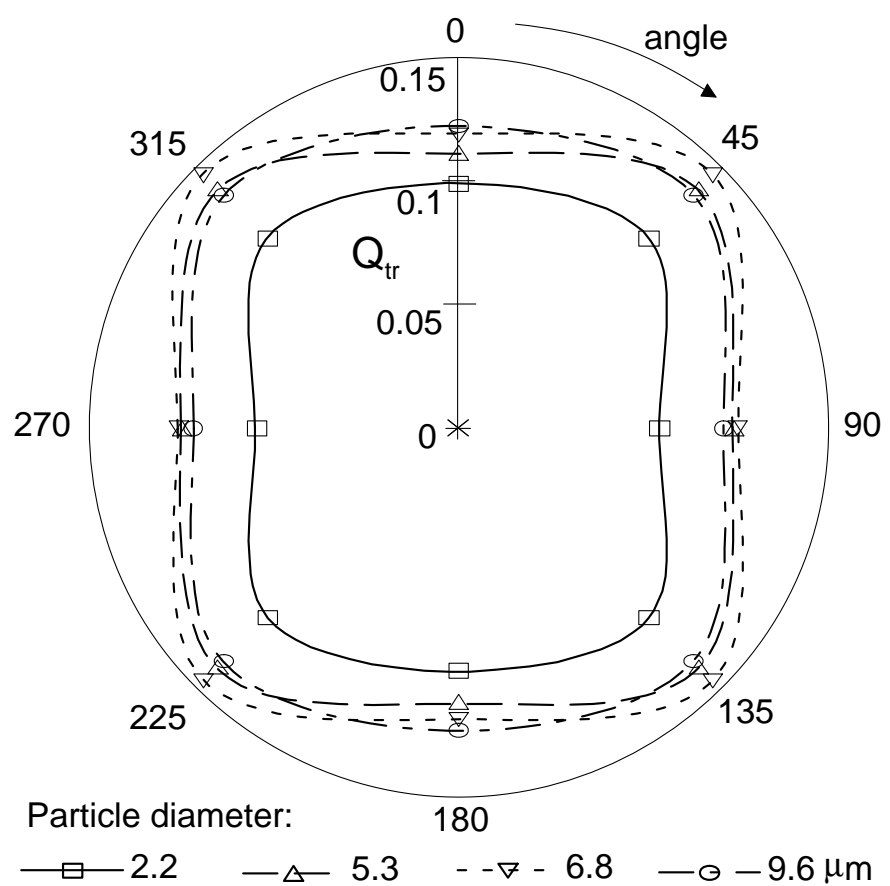

Figure 6. Trapping efficiency as a function of direction of spot movement. The long axis of the focused spot and the direction of polarization are both in the $0^{\circ}$ (vertical) direction.

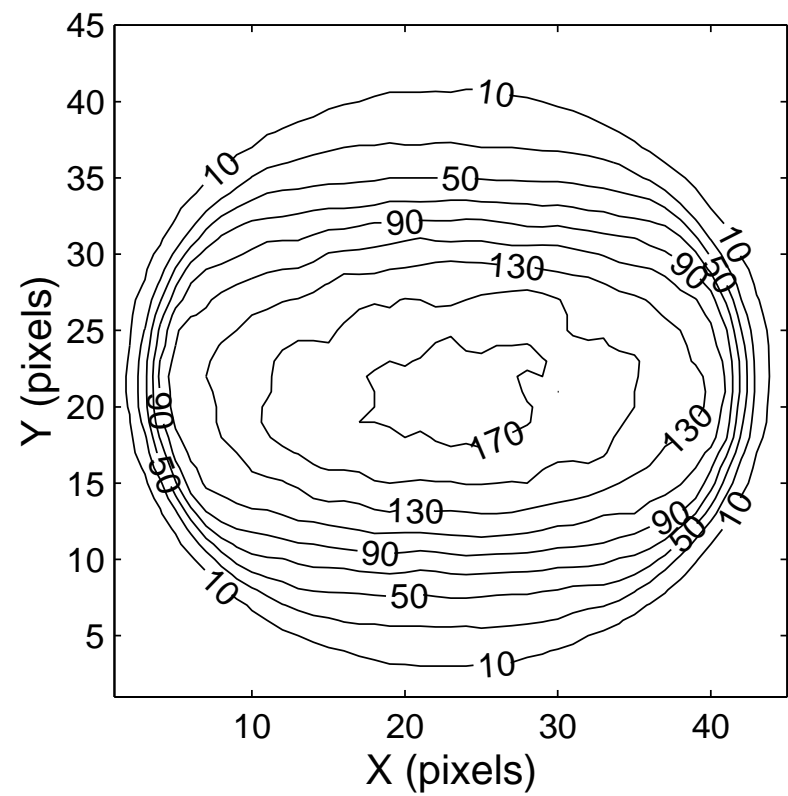

Figure 7. Beam irradiance profile measured prior to focusing at a location corresponding the principal point of the objective. The scale is $143 \mu \mathrm{m}$ per pixel. 


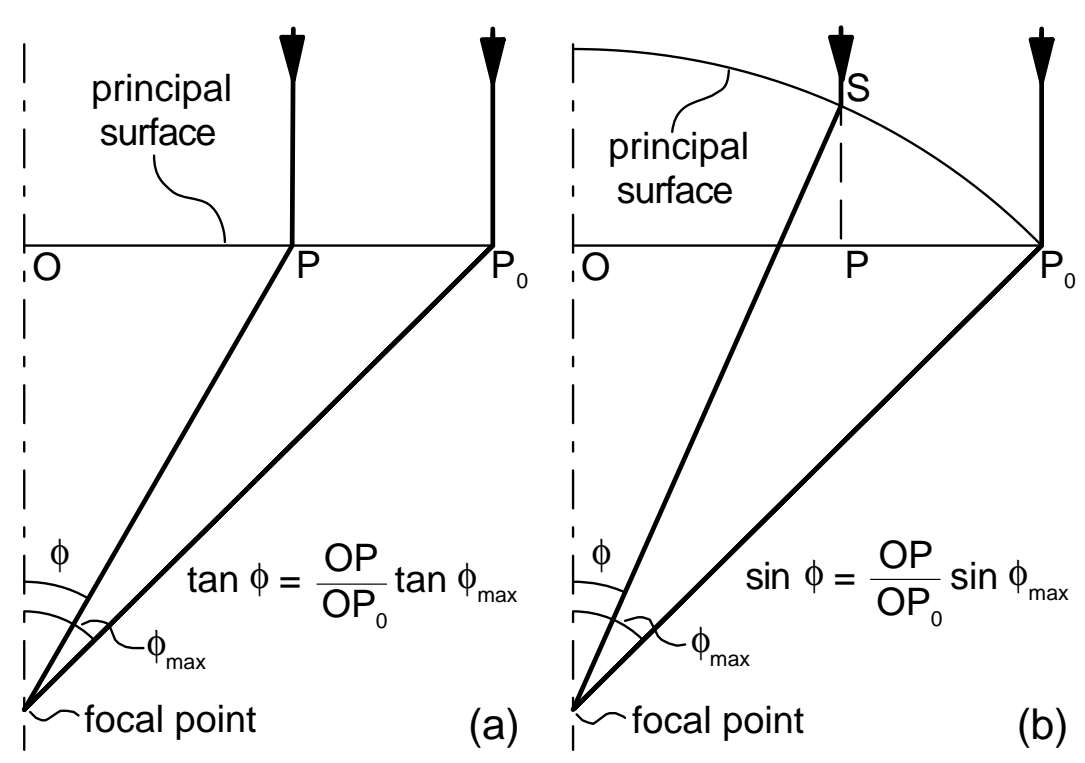

Figure 8. Ray paths for a paraxial lens (a) and a lens satisfying the sine condition (b). In both cases the marginal ray $\mathrm{P}_{0} \mathrm{~F}$ angle $\phi_{\max }$ is dedined by the relationship NA $=n_{0} \sin \phi_{\max }$, where

$\mathrm{NA}$ is the numerical aperture and $n_{0}$ the refractive index of the medium.

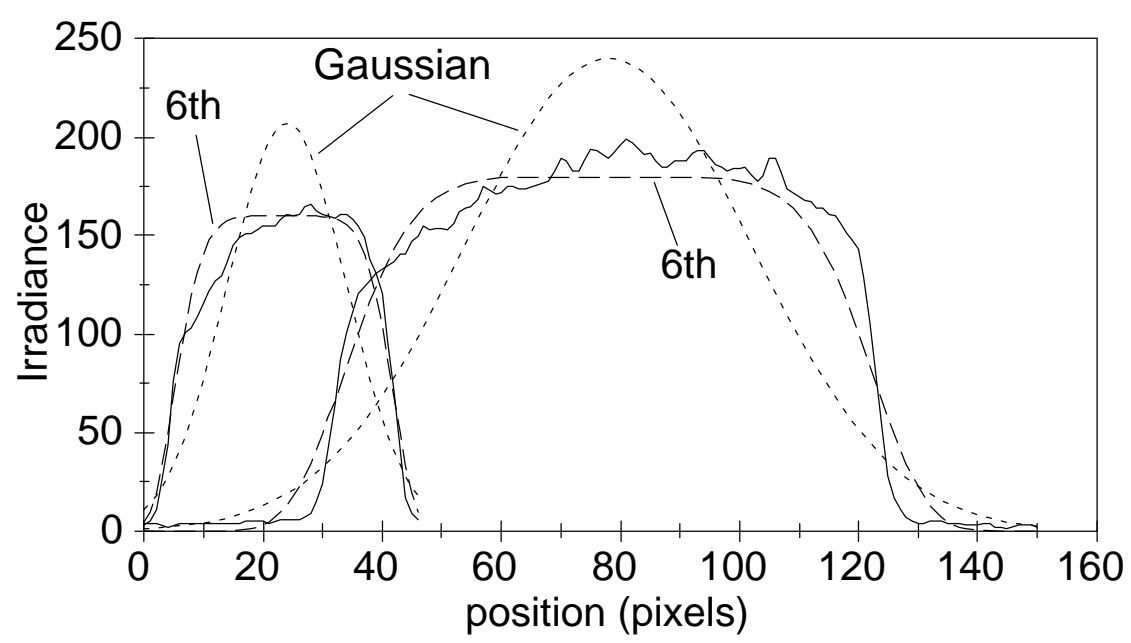

Figure 9. Irradiance profiles measured along the short and the long dimension of the beam (solid line) compared to standard Gaussian function fit (dotted line) and the 6th-power expression (8) (dashed line). The scale is $143 \mu \mathrm{m}$ per pixel. 